\title{
Furniture kits and physical model as a tool for visualization of the minimum residential spaces according to the anthropometric ergonomics
}

\section{SIGRADI2018 TECHNOPOLITICAS \\ xxii congresso da sociedade iberoamericana de gráfica digital 22th conference of the iberoamerican society of digital graphics 07|08|09|novembro|2018 iau usp | são carlos | sp br}

\author{
Luana Peroza Piaia \\ Universidade Comunitária da Região de Chapecó | PósARQ - Universidade Federal de \\ Santa Catarina | Brazil | luanapiaia@unochapeco.edu.br
}

\author{
Alice Theresinha Cybis Pereira \\ PósARQ - Universidade Federal de Santa Catarina | Brazil | acybis@gmail.com
}

Carla Cristina Secchi

Universidade Comunitária da Região de Chapecó | PósARQ - Universidade Federal de

Santa Catarina | Brazil | cah_secchi@unochapeco.edu.br

\begin{abstract}
This paper presents the results obtained through the development of furniture kits and physical model, aiming to compare the minimum design requirements described in the anthropometric ergonomics, current housing program and construction law. The qualitative approach through bibliographical and documentary revision allowed the choice of technical representation, using BIM software for design and subsequent digital prototyping. It concludes that the creation of environments according to the minimum requirements does not allow the use of space according to its purpose, and by making use of furniture kits, it is possible to obtain experiments and better understanding.
\end{abstract}

Keywords: Physical model; Housing of social interest; Rapid prototyping; Minimal spaces; Anthropometric ergonomics.

\section{INTRODUÇÃO}

O elevado crescimento populacional ocorrido no Brasil no final do século XIX, ocasionado pelo êxodo rural, assim como o esgotamento da infraestrutura básica dos centros urbanos, fez com que a classe baixa fosse direcionada para áreas mais periféricas das grandes cidades em habitações baratas. Desta forma, desde 1937, o governo federal, preocupado com o aumento desgovernado da população nas grandes cidades, vêm propondo a criação de programas e planos habitacionais, iniciando a produção estatal de moradias, garantindo o financiamento imobiliário e o surgimento das Habitações de Interesse Social - HIS.

Entretanto, as diferentes políticas habitacionais implementadas pelo governo, agravadas por fatores sociais, culturais e econômicos ao longo dos anos, não resolveram os problemas habitacionais. Como resultado, a produção das HIS no Brasil, em sua maioria, se resume em grandes conjuntos habitacionais destinados às famílias de baixa renda, projetados de forma padronizada, sem planejamento e não provendo o mínimo de conforto, comodidade e funcionalidade, como relata Palermo (2009) "[...] depois de sucessivos programas que, focando a produção desenfreada de unidades de má qualidade construtiva e má qualidade funcional, esqueceram de quem realmente importava, o morador" (p. 13).
A baixa qualidade habitacional faz com que surjam moradias em locais irregulares e onde a coabitação familiar passa a acontecer com maior frequência. Com isso, o déficit habitacional cresce, dificultando a regularização das habitações. Com base na Fundação João Pinheiro (2016), em 2014, foi estimado que 6,068 milhões de unidades residenciais encontravam-se em condições precárias no Brasil.

Nardelli (2014), afirma que no Brasil, o déficit habitacional se configura como um grande desafio social, que perdura por décadas, agravado pelos diversos planos habitacionais, que caracterizados pela lógica e pensamento industrial da repetição, com diminuição de custos construtivos e produção de edificações em larga escala, acabam desconsiderando o aspecto de lar, gerando habitações com baixo nível de conforto em regiões para as quais não foram projetadas, desconsiderando, assim, ambiente e moradores.

É lamentável que o termo "economia" seja o item mais importante quando se fala em HIS, de modo a criar certo descaso para o jeito como são projetados os ambientes para a habitação social. Quando se trata de arquitetura voltada à moradia, os elementos considerados devem ir além dos requisitos e dimensionamentos mínimos prédefinidos (Logsdon, 2012). É preciso projetar ambientes funcionais e flexíveis, que propiciem a melhora na 
qualidade de vida dos usuários, de modo a constituir aquela edificação como lar. De acordo com Palermo (2009), a habitação deve prover um espaço onde as necessidades humanas, como convívio, saúde e crescimento, aconteçam de forma confortável e segura, pois a habitação não é vista pelo usuário somente como um abrigo, mas sim um lar, carregado de sentimentos e simbologias, que conduz seus sonhos.

Mesmo sabendo que a HIS é um recurso para amenizar o elevado percentual do déficit habitacional, ainda há muito o que fazer para que o quadro habitacional seja melhorado e estimulado de outras formas no país. Sabe-se que acabar com o déficit habitacional é algo utópico, mas é preciso rever o grande número de pessoas que moram em condições precárias, e tentar no mínimo projetar ambientes agradáveis, propiciando conforto, melhorando assim a qualidade de vida dos usuários. Nesse aspecto, a ergonomia antropométrica pode se tornar uma aliada, pois prevê em seus estudos a relação do ser humano com o ambiente, de modo a torná-lo mais confortável e funcional (Panero \& Zelnik, 2014), sendo possível delimitar o tamanho dos ambientes e a disposição dos equipamentos, contribuindo na realização das atividades domésticas (lida, 2016).

A ergonomia aborda todos os requisitos relacionados com as atividades que o ser humano realiza, de forma a tornálos mais agradável, prático e seguro. A ergonomia antropométrica está relacionada com as dimensões físicas do corpo humano. Antes de propor qualquer projeto de arquitetura, o projetista deve primeiramente compreender os requisitos propostos pela antropometria, obtendo assim, noções de proporção e escala, de modo que cada ambiente e mobiliário seja projetado de forma coerente e correta para ser utilizado pelo ser humano. Lelis (2015), ainda complementa que a antropometria pode ser definida como a relação entre as medidas humanas e a edificação, pois estas impactam diretamente na habitabilidade do ambiente.

Para auxiliar na concepção de projetos arquitetônicos, as características antropométricas do ser humano devem ser levadas em consideração, pois estas delimitam a questão projetual, identificando a dimensão do espaço como um todo. Para obter melhor apropriação do espaço, busca-se na ergonomia antropométrica, compreender como é definido a dimensão corporal humana, bem como a disposição do mobiliário nos ambientes, espaçamento mínimo necessário para transitar e utilizar o mobiliário, melhorando assim a funcionalidade dos projetos.

Contribuindo de forma eficaz no processo de projeto, as ferramentas computacionais atuam como facilitadoras na tarefa que os projetistas realizam na concepção dos projetos. Conforme salienta Nardelli (2014), as tecnologias digitais aplicadas nas HIS oportunizam a investigação de novas soluções projetuais. Elas estão cada vez mais presentes na arquitetura, instigando a criatividade e produção arquitetônica (Vanzin, Bastiani, \& Almeida, 2015). Seu uso permite uma maior personalização, eficiência, economia, flexibilidade e adaptação (Cruz, 2016). Nesse sentido, a utilização de ferramentas digitais de projeto, permite possibilidades de experimentações. Destacam-se no presente estudo o BIM (Building Information Modeling) e a prototipagem digital.
Com a tecnologia BIM é possível integrar todas as etapas e responsáveis pelo processo de projeto em um único arquivo. Promove a coordenação das atividades de projeto, a integração de informações e a compatibilização de dados desde a concepção até a fase final de projeto.

Em relação à prototipagem digital, esta atua como ferramenta de apoio ao processo de projeto, possibilitando a criação precisa de maquetes físicas, com arquivos digitais enviados diretamente aos maquinários de controle numérico (Nardelli, 2014), comprovando que o protótipo possibilita melhor comunicação e entendimento do projeto, que talvez não fosse possível ser evidenciado somente na representação digital (Pupo, 2009). Estes protótipos são utilizados em diversos momentos na criação do projeto, dentre os quais no estudo inicial e final de uma proposta, análise de objetos/ mobiliário em relação a ergonomia dos mesmos, avaliação final de cada objeto/ produto criado (Bruscat, Brendler, Viaro, Teixeira, \& Silva, 2013).

De acordo com Pupo (2009) o termo prototipagem digital engloba todos os processos de prototipagem rápida, assim como o corte a laser, corte/desbastes com fresas e corte em vinil, que compreendem a produção de maquetes e protótipos em escalas reduzidas.

O BIM e a prototipagem digital já estão há muitos anos no mercado (Nardelli, 2014), e vários casos de sua utilização em HIS podem ser citados. Como exemplo, tem-se a pesquisa de Nardelli (2010), Nardelli (2014) e de Nardelli e Backheuser (2016) que investigam o uso da prototipagem e fabricação digitais, do BIM e da arquitetura generativa, na busca de soluções para o programa minha casa minha vida - PMCMV, desenvolvendo protótipos e peça finais de construção, propondo novas tipologias arquitetônicas. Outro caso é a pesquisa de Mendes (2014), que propôs um método de implantação de HIS, através da gramática da forma. Porém, ainda há muito a ser explorado.

Esse avanço tecnológico representa a atual necessidade de mudanças que o campo da construção civil necessita atualmente. A implementação de tecnologias, que venham a auxiliar na concepção dos projetos, mostra que é necessário rever as técnicas utilizadas na construção civil brasileira, pois estão ultrapassadas em termos globais (Nardelli, 2014).

Desta forma, o presente artigo apresenta o desenvolvimento de kits de mobiliários para visualização do aspecto de distanciamento e layout dos mesmos em ambiente interno de HIS. Utiliza-se o BIM e a prototipagem digital como instrumentos de desenvolvimento dos kits para compreender e comparar os requisitos mínimos de projeto, exigidos nas HIS, e o que é recomendado pela ergonomia antropométrica. Importante colocar aqui que embora o BIM promova o 3D, os aspectos que se pretende enfatizar com este material são melhor visualizados em 2D, por isso os Kits são desenvolvidos nesta dimensão.

\section{METODOLOGIA}

A pesquisa possui caráter qualitativo, com método experimental e indutivo, baseado em comparação, seguindo as seguintes etapas, conforme figura 01 : 


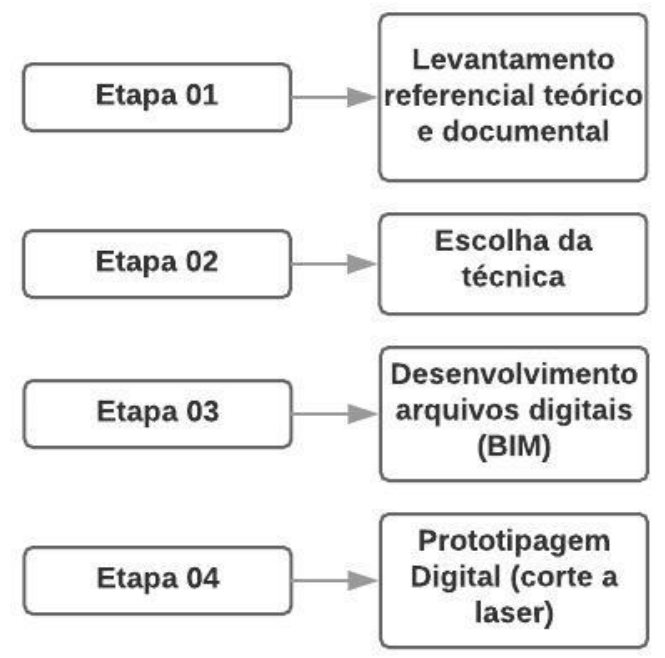

Figura 01: Etapas metodológicas. Fonte: Autores.

\section{ETAPA 01}

Na primeira etapa do estudo foi realizado o levantamento do referencial teórico e documental sobre HIS e ergonomia antropométrica. Com estes levantamentos realizados, buscou-se identificar e analisar os espaços mínimos necessários para cada ambiente interno de uma residência de acordo com a antropometria, para após realizar a comparação destes com os requisitos mínimos dos ambientes estabelecidos no programa minha casa minha vida (PMCMV) e no código de obras municipal de Chapecó, tendo em vista que os projetistas se baseiam nestas leis para a realização dos projetos habitacionais. Além das dimensões dos ambientes mínimos, foram identificadas as circulações, espaços de circulação e uso necessários para a utilização dos mobiliários estabelecidos de cada um dos três quesitos de comparação.

Para isso foram levantadas as medidas mínimas que constam no PMCMV, código de obras municipal e na ergonomia antropométrica dos seguintes ambientes residenciais: sala de estar, sala de jantar, cozinha, área de serviço, dormitório de casal, dormitório de solteiro e banheiro.

\section{ETAPA 02}

Com a pesquisa inicial realizada e os dados dos ambientes e espaços mínimos levantados, passou-se para a escolha das técnicas passíveis de representação e visualização. Em um primeiro momento, viu-se a necessidade de representação dos dados digitalmente, em forma de desenho em planta baixa. Como os autores do presente estudo já possuem domínio em software BIM, este foi o meio de representação digital escolhido, além das vantagens que o uso do BIM possibilita. Apesar de já possível visualização de inconsistências nos desenhos digitais, identificou-se a prototipagem digital como uma técnica que permitiria a compreensão dos espaços de outra forma, com possibilidade de manuseio e experimentações, pois de acordo com Batistello, Balzan, Piaia, \& Miotto (2015), os protótipos como materialização e representação física de um modelo digital permitem a clareza da proposta e a exata avaliação, agregando na criação projetual com possibilidade de produção rápida de diferentes opções, e esse é o grande diferencial com relação aos arquivos digitais, afirmam os autores, pois auxilia no entendimento e percepção da proposta.

Assim, passou-se para a abordagem através das tecnologias digitais, para realização dos estudos, pois possibilitam a produção digital e física de modo a permitir a realização da comparação dos requisitos mínimos levantados.

\section{ETAPA 03}

Na terceira etapa, deu-se início ao desenvolvimento dos desenhos, em software BIM. Foram realizados os projetos dos ambientes internos mínimos para uma habitação social, além dos espaços mínimos de circulação e uso do mobiliário conforme os dados obtidos na etapa 01. Com isso, busca-se compreender qual o efeito que tais dimensões mínimas estipuladas pela antropometria, programa MCMV e código de obras municipal ocasionam em uma residência de HIS.

Ainda que o software BIM utilizado permita o desenho em $3 \mathrm{D}$, os arquivos desenvolvidos foram feitos em 2D, pois é desta forma, em duas dimensões, que melhor representa os constrangimentos encontrados. Os arquivos foram desenvolvidos da seguinte forma: foi realizado uma tabela comparativa, com a área mínima de cada ambiente interno de acordo com os três requisitos deste estudo (PMCMV, Código de obras e antropometria), conforme mostra a Tabela 01.

Tabela 1: Especificação da área mínima total de cada ambiente habitacional, de acordo com as temáticas levantadas

\begin{tabular}{|c|c|c|c|}
\hline \multirow{2}{*}{ AMBIENTE } & \multicolumn{3}{|c|}{ ÁREA TOTAL } \\
\cline { 2 - 4 } & $\begin{array}{c}\text { Ergonomia } \\
\text { Antropomé - } \\
\text { trica }\end{array}$ & $\begin{array}{c}\text { Programa } \\
\text { Minha Casa } \\
\text { Minha Vida }\end{array}$ & $\begin{array}{c}\text { Código de } \\
\text { Obras } \\
\text { municipal }\end{array}$ \\
\hline Sala de Estar & $3,75 \times 3,55$ & $2,40 \times 2,40$ & $2,50 \times 2,50$ \\
\hline $\begin{array}{c}\text { Sala de } \\
\text { Jantar }\end{array}$ & $2,26 \times 3,55$ & $2,40 \times 2,40$ & $2,50 \times 2,50$ \\
\hline Cozinha & $2,42 \times 2,94$ & $1,80 \times 2,60$ & $1,80 \times 1,80$ \\
\hline $\begin{array}{c}\text { Área de } \\
\text { Serviço }\end{array}$ & $1,72 \times 2,24$ & $1,50 \times 1,20$ & $1,20 \times 1,20$ \\
\hline $\begin{array}{c}\text { Dormitório } \\
\text { Casal }\end{array}$ & $3,60 \times 3,65$ & $2,40 \times 3,00$ & $2,50 \times 2,50$ \\
\hline $\begin{array}{c}\text { Dormitório } \\
\text { Solteiro }\end{array}$ & $2,71 \times 3,66$ & $2,40 \times 3,00$ & $2,50 \times 2,50$ \\
\hline Banheiro & $1,26 \times 2,84$ & $1,50 \times 2,30$ & $1,20 \times 1,20$ \\
\hline
\end{tabular}

Foram desenhados juntamente com os mobiliários, as circulações/espaços mínimos para uso do espaço e das atividades no ambiente. Esse dado foi retirado dos estudos realizados sobre a ergonomia antropométrica.

Para propor a realização dos kits, optou-se em desenhar a área mínima dos ambientes residenciais em três 
momentos: $1^{\circ}$ como solicita a ergonomia antropométrica; $2^{\circ}$ como indica o PMCMV e por $3^{\circ}$ como é proposto pelo código de obras. Foram colocadas uma ao lado da outra, como forma de comparação.

Com o projeto realizado no BIM, já foi possível identificar as grandes inconsistências e problemas encontrados na concepção de cada ambiente, porém, a visualização somente digital não foi capaz de reproduzir o que a percepção física que uma maquete proporciona. Através desta conclusão e após a criação dos ambientes em software BIM, passou-se a preparação dos mesmos para a realização da prototipagem digital, conforme mostra a figura 02.

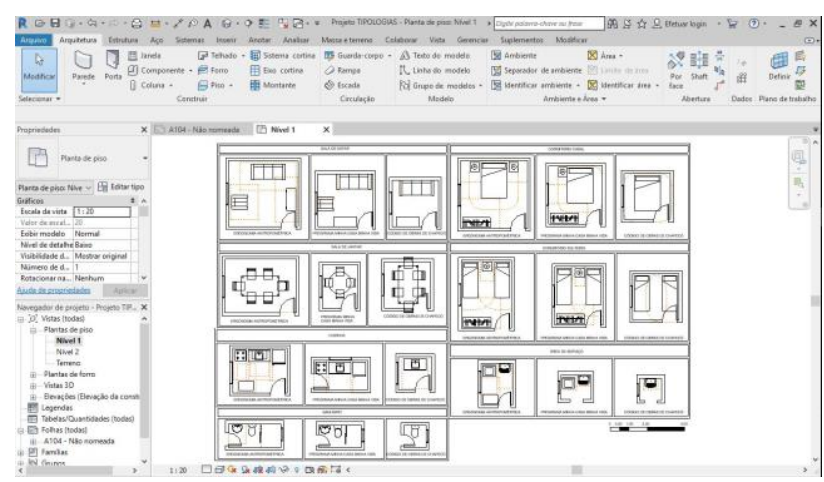

Figura 02: Ambientes, kit de mobiliário e espaços de circulação e uso desenvolvidos no software BIM. Fonte: Autores.

\section{ETAPA 04}

A técnica de prototipagem digital escolhida para o desenvolvimento da quarta etapa, que é a prototipagem dos kits de mobiliário e maquetes físicas dos ambientes, foi o corte a laser, devido ao acesso (foi utilizado o maquinário de um FABLAB - Fabrication laboratory, implantado em uma universidade), custo e resultado.

Desta forma, foram prototipados os kits de mobiliário e maquetes físicas dos ambientes desenvolvidos no software BIM, conforme relata a etapa 03 . As maquetes físicas compreendem os ambientes mínimos apontados pelo PMCMV, ergonomia antropométrica e código de obras de Chapecó. Já os kits, compõe-se do mobiliário mínimo necessário para a utilização em cada ambiente, sendo esta parte fundamental na organização espacial do espaço residencial.

Após a prototipagem dos kits de mobiliário e maquetes dos ambientes, viu-se a necessidade de prototipar um kit com os distanciamentos mínimos de mobiliário e ou equipamento de cada ambiente, representando seu uso e circulação necessários. Para isso foi realizado o desenho destes espaços no BIM e após realizada a prototipagem. A Figura 03 demonstra o momento em que está sendo realizado a prototipagem desta etapa através do corte a laser, assim como o resultado final dos kits de mobiliários e espaços de uso e circulação.
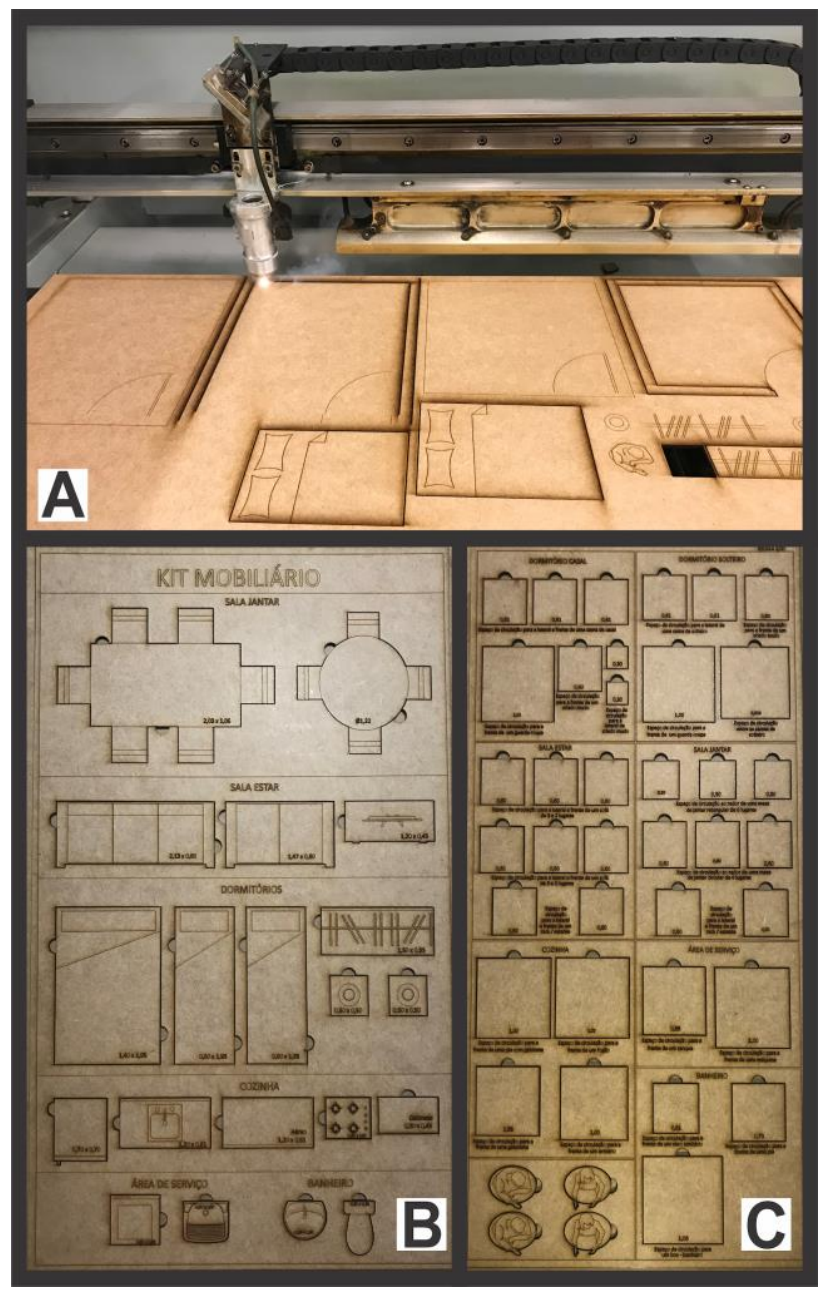

Figura 03: A - Prototipagem em uma máquina de corte a laser; B Kit dos mobiliários; C - Kit dos espaços de uso e circulação. Fonte: Autores.

\section{RESULTADOS}

Foi possível verificar após o desenvolvimento das maquetes e kits de mobiliário e espaços de uso e circulação que os requisitos mínimos descritos pelo PMCMV e código de obras de Chapecó não permitem utilizar os ambientes de uma HIS de acordo com suas funções e estão bem distantes do que é desejável pela ergonomia antropométrica. Os protótipos confeccionados permitem o manuseio e a percepção física, que instiga uma sensibilidade e entendimento diferente daquela vista somente no ambiente virtual.

A comparação entre os requisitos mínimos em relação a área de cada ambiente, estipulados em cada temática, fica melhor compreensível com a utilização das ferramentas computacionais utilizadas para realizar este estudo. A Figura 04 apresenta os ambientes, juntamente com a distribuição do mobiliário e espaços de uso e circulação prototipados. 


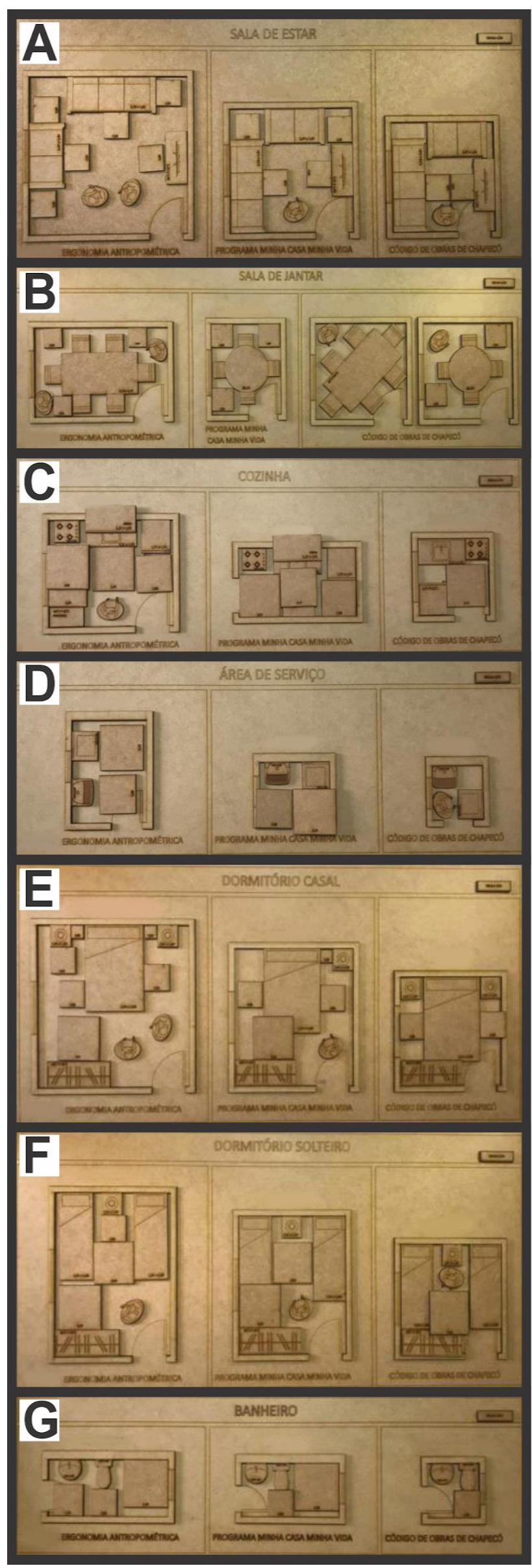

Figura 04: Maquetes comparativas dos ambientes de HIS de acordo com o PMCMV, a Ergonomia Antropométrica e o Código de obras de Chapecó, dos seguintes ambientes: A - Sala de estar; B - Sala de Jantar; C - Cozinha; D - Área de serviço; E Dormitório casal; F - Dormitório solteiro; G - Banheiro. Fonte: Autores.

Com o uso das ferramentas computacionais, foi possível observar que os ambientes projetados de acordo com o requisito mínimo de área descrito pelo programa habitacional e código de obras não conseguem receber o mobiliário mínimo para cada ambiente, isso faz com que surjam questionamentos acerca de como foram estipuladas estas dimensões mínimas que os mesmos solicitam na realização de projetos para HIS.

Além de verificar a inconstância nas dimensões, as maquetes prototipadas permitem experimentações de diferentes layouts através dos kits de mobiliários e dos espaços de circulação e uso mínimo necessário. Com a prototipagem foi possível compreender melhor como é a concepção de cada ambiente, além de perceber que em muitos casos o distanciamento entre mobília e parede é insuficiente. Por isso, é importante que os projetistas compreendam o que a ergonomia antropométrica propõe em relação aos requisitos mínimos para cada ambiente, de modo a obter espaços adequados e planejados conforme a necessidade de cada usuário.

\section{DISCUSSÃO}

O presente estudo contribui para a compreensão de como os ambientes internos em HIS estão sendo projetados hoje em dia e a importância da ergonomia antropométrica no momento de projetar. A prototipagem digital auxilia no estudo como uma ferramenta de auxílio muito importante na percepção e visualização dos espaços dentro do processo de projeto, proporcionando uma outra forma de compreensão e análise dos espaços.

A existência de incoerências entre indicações constantes apresentadas no código de obras quando comparados aos preceitos da ergonomia antropométrica induzem a erros projetuais, como pôde ser observado nos ambientes desenhados. Já o PMCMV está mais de acordo com o que propõe a antropometria, mas também é preciso rever as dimensões mínimas solicitadas pela normativa.

Para isso, é preciso que o modo como são projetados os ambientes para HIS sejam revistos, pois de nada adianta propor uma edificação se o usuário não puder usufruir de todos os ambientes de modo ergonômico, propiciando conforto e bem-estar.

Por isso considera-se importante que a academia seja o local para alertar sobre estas incoerências, motivando reflexões projetuais, de modo a incentivar o futuro profissional a pensar melhor na concepção de cada ambiente interno. É preciso que o problema habitacional seja visto como algo desafiador, pois sabe-se que é impossível acabar com o déficit habitacional, mas com o auxílio das ferramentas computacionais, é possível repensar o modo como a arquitetura para a habitação de interesse social está sendo proposta.

Com a materialização dos ambientes modelados no BIM por meio da prototipagem realizada através do corte a laser, foi possível ter uma nova percepção das formas dos 
ambientes de modo palpável. Isso possibilita também, um novo método de reorganização imediata do mobiliário. A modelagem pelo BIM e prototipagem estimula a reflexão e compreensão dos ambientes internos, ficando aqui a sugestão de inserção desses métodos no meio acadêmico, proporcionando melhor apropriação e percepção do projeto como um todo, pois com o BIM são analisados os espaços de circulação e uso que se sobrepõem nas paredes e mobiliário (conforme mostra a Figura 02) e pela prototipagem por não encaixar no ambiente já proposto, sendo estes os diferenciais que essas tecnologias propiciam, contribuindo de todas as formas na tomada de decisão no processo de projeto.

\section{REFERÊNCIAS}

Batistello, P., Balzan, K. L., Piaia, L. P., \& Miotto, J. (2015). Prototipagem rápida e fabricação digital em ateliê vertical: do processo à materialização Paper presented at the XIX Congresso da Sociedade Ibero-Americana de Gráfica Digital Sigradi, Florianópolis.

Bruscato, U. M., Brendler, C. F., Viaro, F. S., Teixeira, F. G., \& Silva, R. P. d. (2013). Uso da Fabricação Digital e Prototipagem Rápida no Desenvolvimento do Projeto de Produto: Análises do Produto através de Simulações Digitais. Paper presented at the XVII Conference of the Iberoamerican Society of Digital Graphics - SIGraDi, Valparaíso. http://pdf.blucher.com.br.s3-sa-east-

1.amazonaws.com/designproceedings/sigradi2013/0088.pdf

Cruz, N. L. e. (2014). Da produção à customização em massa: parametrização e desenvolvimento do sistema construtivo Wikihouse. (Mestrado), Universidade de Lisboa, Lisboa. Retrieved from https://repositorio.iscteiul.pt/handle/10071/13196 (201551764)

lida, I. (2016). Ergonomia: projeto e produção (3 ed.). São Paulo: Blucher.

Lelis, V. F. (2015). Análise de habitações sociais na perspectiva da ergonomia do ambiente construído: estudos de caso do PROMORE. (Mestrado), Universidade Estadual Paulista "Júlio de Mesquita Filho", Bauru. Retrieved from https://repositorio.unesp.br/handle/11449/136659
Logsdon, L. (2012). O Programa Minha Casa Minha Vida em Cuiabá-MT: uma análise da qualidade dos projetos destinados às famílias de baixa renda. (Mestrado), Universidade Federal de Santa Catarina - UFSC, Florianópolis. Retrieved from https://repositorio.ufsc.br/xmlui/handle/123456789/100364

Mendes, L. T. (2014). Personalização de habitação de interesse social no Brasil : o caso da implantação urbana em conjuntos habitacionais. (Doutorado), Universidade Estadual de Campinas - UNICAMP, Campinas. Retrieved from http://repositorio.unicamp.br/jspui/handle/REPOSIP/258031

Nardelli, E. S. (2010). Tecnologia digital avançada na produção de Habitações de Interesse Social - HIS no Brasil. Paper presented at the XIV Congreso de la Sociedad Iberoamericana de Grafica Digital - Sigradi, Bogotá.

Nardelli, E. S. (2014). Fabricação Digital na Produção de Habitação de Interesse Social no Brasil. Paper presented at the Congreso de la Sociedad Iberoamericana de Gráfica Digital - Sigradi, Uruguay.

Nardelli, E. S., \& Backheuser, L. A. F. (2016). Sistema Wikihouse aplicado ao Programa Minha Casa Minha Vida. Paper presented at the XX Congreso de la Sociedad Iberoamericana de Gráfica Digital - Sigradi, Buenos Aires. http://papers.cumincad.org/data/works/att/sigradi2016_461.p df

Palermo, C. (2009). Sustentabilidade social do habitar. (1 ed.). Florianópolis: Oficina De Textos.

Panero, J., \& Zelnik, M. (2014). Dimensionamento humano para espaços interiores: um livro de consulta e referência para projetos. Barcelona: GG.

Pinheiro, F. J. (2012). Déficit habitacional no Brasil 2013-2014. Belo Horizonte: Fundação João Pinheiro Retrieved from http://www.fjp.mg.gov.br/index.php/docman/cei/informativoscei-eventuais/634-deficit-habitacional-06-09-2016/file

Pupo, R. (2009). Inserção da PROTOTIPAGEM e FABRICAÇÃO DIGITAIS no processo de projeto: um novo desafio para o ensino de arquitetura. (Doutorado), Universidade Estadual de Campinas, Campinas.

Vanzin, T., Bastiani, J. D., \& Almeida, L. O. D. (2015). Criatividade e inovação: a impressão 3D no ensino de arquitetura. In Criatividade e Inovação na educação (1 ed., pp. 115-145). São Paulo: Pimenta Cultural. 